\title{
UNA VIGILIA DI INCERTEZZE
}

\author{
di Giacomo Sani
}

In tutte le democrazie i mesi che precedono una scadenza elettorale sono caratterizzati da un certo grado di incertezza che, a seconda delle particolarità del sistema politico, può riguardare il nome del vincitore, il margine della vittoria (o della sconfitta) di questo o di quel gruppo politico, il raggiungimento da parte di un cartello elettorale della maggioranza dei seggi nelle assemblee legislative, o le conseguenze del verdetto per le policies del futuro governo. A posteriori l'esito di una consultazione qualche volta appare scontato; ma basta ricollocarsi nell'atmosfera delle settimane e dei mesi che precedono il voto per rendersi conto del clima di suspense, dei timori e delle speranze con i quali protagonisti, osservatori e cittadini vivono la vigilia.

Naturalmente, il grado di incertezza varia in parte in funzione delle circostanze e dei contesti. Su di esso influiscono infatti una varietà di elementi quali la percezione dell'importanza della posta in palio, la natura delle issues, l'asprezza dello scontro, il livellamento delle forze in campo, le influenze provenienti dal contesto internazionale ed altre ancora. L'incertezza si riduce, ma non sparisce mai del tutto, mano a mano che ci si avvicina alla scadenza, si definiscono le posizioni dei gruppi in competizione, e si cristallizzano gli orientamenti del corpo elettorale.

Ma al di là degli effetti prodotti dalle particolarità di una data congiuntura o di un dato contesto istituzionale, il grado di incertezza di ogni vigilia elettorale dipende da tre fattori di carattere generale e cioè:

(a) la continuità o meno delle regole elettorali;

(b) la configurazione dell'offerta politica in termini di schieramento, di proposte, di personale;

(c) la risposta dell'elettorato alle proposte dei gruppi in competizione nel quadro dei vincoli e delle opportunità offerti dalle regole. 
È importante sottolineare che questi tre fattori non agiscono indipendentemente ma sono collegati in parte tra di loro in una struttura gerarchica, o «ad imbuto». La natura dell'offerta dipende ovviamente anche dalle regole; a sua volta, la risposta dei cittadini dipende sia dalla loro interpretazione dei vincoli che scaturiscono dalle regole che dalle loro percezioni dell'offerta proveniente da parte dei gruppi in competizione.

Il massimo dell'incertezza si ha nelle transizioni da un regime autoritario ad uno democratico perché in questi passaggi da sistema a sistema esistono grosse incognite relativamente a tutti e tre gli elementi di cui sopra. Le regole sono per definizione nuove e non se ne conoscono appieno gli effetti; l'offerta è di solito poco strutturata e rappresenta una grossa incognita; $e$, infine, non vi sono precedenti che consentano di stimare con una certa affidabilità gli orientamenti dell'elettorato. Conversamente l'incertezza è ridotta al minimo nei sistemi in cui la competizione è fortemente istituzionalizzata, non vi sono cambiamenti nelle regole elettorali, il panorama delle forze in campo rimane sostanzialmente stabile, e le precedenti esperienze indicano che la risposta della cittadinanza alle proposte dei partiti varia solo entro limiti ristretti.

Dal punto di vista del grado di incertezza la maggior parte delle vigilie elettorali si avvicinano più al secondo che al primo di questi estremi. I mutamenti delle regole sono infatti eventi relativamente poco frequenti, i sistemi di partito sono tendenzialmente stabili e producono solo variazioni minori dell'offerta elettorale, e, nonostante l'avvicendarsi delle generazioni, a livello di massa tra una consultazione e un'altra si riproducono nell'aggregato orientamenti solo marginalmente diversi. Nel corso dei primi quarantacinque anni della storia elettorale italiana le regole sono mutate solo nel 1953 (ma senza produrre effetti) e, molto marginalmente, nel 1992 con la modifica relativa al voto di preferenza. Il quadro complessivo delle forze in campo è rimasto per decenni in larga misura invariato, anche se non sono mancate in alcune occasioni le novità, e la dinamica degli orientamenti del corpo elettorale è stata, nel complesso, piuttosto lenta. Viste nella prospettiva di lungo periodo, le variazioni delle quote di consenso ricevute dalle diverse formazioni politiche italiane lungo l'arco di diversi decenni appaiono assai modeste.

Rispetto a questo quadro di sostanziale stabilità, la consultazione del marzo 1994 rappresenta un momento di rottura ri- 
spetto a tutte e tre le variabili indicate sopra. Si è votato con nuove regole, si è profondamente modificata l'offerta politica con la sparizione di alcuni vecchi protagonisti e l'affacciarsi alla ribalta di nuovi competitori, e, infine, gli elettori hanno risposto in maniera solo in parte riconducibile agli schemi del passato.

Nelle pagine che seguono mi propongo di illustrare gli elementi che hanno contribuito a rendere particolarmente incerta la vigilia dell'ultima consultazione esaminando le modifiche apportate al quadro istituzionale, la dinamica dei comportamenti elettorali nei test delle elezioni amministrative e la trasformazione del sistema dei partiti dovuta all'emergere di una configurazione della offerta significativamente diversa da quelle del passato.

Le regole: l'anno della svolta

Il 1993 verrà ricordato nella storia elettorale italiana come un anno particolarmente importante e senza precedenti nella vita della repubblica. In esso si ha infatti una forte accelerazione del ciclo politico-elettorale iniziatosi con le regionali del 1990 e proseguito attraverso le tappe del referendum 1991 sulla preferenza unica, delle elezioni politiche del 6 aprile 1992, e di alcuni test elettorali in ambito locale limitato, ma politicamente significativi, tenutisi nell'autunno dello stesso anno.

Per rendersi conto della importanza delle trasformazioni avvenute nel 1993 è utile ricordare brevemente i provvedimenti legislativi e gli appuntamenti elettorali intervenuti nell'arco di soli dieci mesi (da marzo a dicembre). A fine marzo il parlamento licenziava una nuova disciplina per l'elezione degli organi amministrativi di comuni e province ${ }^{1}$. Si trattava di una normativa fortemente innovatrice rispetto alle regole del passato e che prevedeva istituti del tutto estranei alla tradizione italiana del dopoguerra quali l'elezione diretta del sindaco e del presidente della provincia, la possibilità di ricorso al ballottaggio, l'attribuzione di un premio di maggioranza alla lista (o liste) collegate col candidato vincente, la netta separazione dei ruoli del consiglio e della giunta, ed altre ancora.

A distanza di tre sole settimane il verdetto del referendum

${ }^{1}$ Le nuove norme sono contenute nella Legge n. 81 del 25-3-93. 
sulla legge elettorale del Senato apriva la strada alle modifiche delle regole del gioco per l'elezione dei due rami del parlamento. Con il 18 aprile il «sì» dell'elettorato all'abolizione del quorum del $65 \%$ per l'elezione dei senatori nei collegi introduceva nella legislazione il principio del maggioritario. Ma poiché i collegi allora esistenti erano 238 e i seggi senatoriali 315 , l'esito del referendum veniva anche automaticamente a fissare in 3 a 1 il rapporto tra quota maggioritaria e quota proporzionale dei seggi. Se il referendum aveva dato una spallata al vecchio sistema, la riforma non poteva tuttavia dirsi completa. Intanto perché il verdetto del 18 aprile introduceva una forte asimmetria tra i metodi di elezione delle due assemblee visto che l'esito del referendum non aveva alcun effetto sul sistema in vigore per la $\mathrm{Ca}$ mera. E poi perché nel caso del Senato la configurazione dei collegi, i cui confini erano rimasti invariati per decenni, presentava forti squilibri sul piano demografico al punto da renderla incompatibile con il principio dell'eguaglianza del voto sancito dall'articolo 48 della Costituzione. Il completamento della riforma richiedeva, dunque, un intervento del legislatore che rendesse operativi per le due assemblee i principi di fondo scaturiti dal referendum.

Il cammino parlamentare risultava alquanto accidentato sia per la diversità di punti di vista tra i diversi gruppi politici e all'interno dei medesimi, sia per gli orientamenti non collimanti presenti nelle due assemblee, sia infine per la resistenza passiva opposta da alcuni settori preoccupati di un eventuale rapido scioglimento delle camere una volta completata la riforma. A complicare le cose contribuivano spinte, contro-spinte e sospetti sull'effettiva capacità di un parlamento almeno parzialmente delegittimato. Ne risultava una sorta di ping-pong del progetto Mattarella che veniva più volte modificato dalle competenti commissioni di Camera e Senato e concludeva, infine, il suo travagliato cammino il 4 agosto con l'approvazione delle leggi n. 276 e n. 277 . Entrambe recepivano il sistema misto maggioritario-proporzionale, con le quote fissate dall'esito del referendum, ma si differenziavano sotto alcuni importanti profili, in particolare quelli relativi alle modalità di assegnazione della quota proporzionale dei seggi e dei meccanismi dello 'scorporo' dei voti conseguiti dai candidati eletti nei collegi uninominali'.

2 Camera dei Deputati (1994). Una approfondita analisi del nuovo sistema elettorale è contenuta in D'Alimonte e Chiaramonte (1993). 
Prima dell'inizio della tradizionale pausa estiva il disegno del nuovo sistema elettorale era finalmente completo: il nuovo parlamento sarebbe stato eletto, a tempi più o meno ravvicinati, con regole del tutto inedite. Un grosso elemento di incertezza era dunque stato rimosso, ma rimanevano in sospeso alcuni punti non irrilevanti. Il primo riguardava la concessione del diritto di voto degli italiani all'estero, un provvedimento che richiedeva una legge costituzionale e che quindi dilatava i tempi per il completamento della riforma. Questo nodo veniva risolto solo nel tardo autunno quando in seconda lettura il provvedimento non otteneva la prescritta approvazione.

Il secondo punto rimasto in sospeso era quello relativo alla determinazione dei confini dei nuovi collegi elettorali uninominali (475 per la Camera e 232 per il Senato) previsti dalle nuove norme. Queste avevano individuato le unità primarie in 27 nuove circoscrizioni per la Camera e nelle 20 regioni per il Senato e avevano altresì fissato $i$ criteri per il ritaglio dei collegi al loro interno. Questa operazione poteva venir considerata un adempimento tecnico, di minore importanza, ma era in realtà un tassello indispensabile senza il quale sarebbe stato impossibile far funzionare il nuovo sistema ${ }^{3}$. Dal punto di vista poi delle forze politiche che andavano preparandosi ad uno scontro elettorale a tempi ravvicinati, il fatto che la mappa dei collegi fosse ancora da disegnare costituiva una grossa incognita e al tempo stesso una fonte di preoccupazione per le insidie potenzialmente rappresentate dal ricorso ( $\mathrm{da}$ parte di altri, naturalmente) alle malizie del gerrymandering.

Le nuove norme avevano assegnato il ritaglio dei collegi ad una commissione di esperti, nominata di concerto dai presidenti dei due rami del parlamento, che iniziava i suoi lavori a fine agosto e completava il disegno della nuova mappa elettorale entro ottobre. La parola passava poi ai consigli regionali cui spettava di esprimere un parere (non vincolante) sulle proposte della commissione di esperti ed infine alle commissioni parlamentari ed all'esecutivo. La versione definitiva della configurazione dei collegi veniva resa nota solo a dicembre avanzato con un decreto legislativo che recependo le osservazioni di alcune re-

${ }^{3}$ Era la stessa normativa approvata il 4 agosto a prevedere che fino alla definizione dei nuovi collegi elettorali sarebbe rimasto in vigore il vecchio sistema (art. $10 \mathrm{~L}$. 277). 
gioni e del parlamento apportava una serie di modifiche al piano originario ${ }^{4}$.

Infine, per completare questa breve rassegna dei cambiamenti apportati alle regole elettorali va ricordato che a metà dicembre il parlamento approvava una legge sulla disciplina delle campagne elettorali che conteneva importanti novità soprattutto nei capitoli relativi alla utilizzazione dei mass media, alla pubblicazione dei sondaggi, al tetto delle spese elettorali ed altri ancora ${ }^{5}$.

Ripercorrendo il cammino fatto da marzo a dicembre non vi è dubbio che le modifiche apportate alla legislazione elettorale abbiano costituito una delle componenti più importanti della cosiddetta «rivoluzione» italiana.

\section{I test di giugno e novembre}

Un secondo elemento di rottura rispetto al passato è rappresentato dalla dinamica dei comportamenti di massa. Ai primi di maggio, mentre iniziava il dibattito sulle implicazioni del referendum, l'attenzione delle forze politiche era in buona parte assorbita dall'inizio della campagna per la prima tornata di elezioni amministrative regolate dalla nuova legge, tornata fissata per il 6-21 giugno che interessava 1.192 consigli comunali (di cui 112 con popolazione superiore ai 15.000 abitanti), sei consigli provinciali ed i consigli regionali del Friuli-Venezia Giulia e della Valle d'Aosta. A rendere particolarmente significativo questo test contribuivano, oltre alla novità delle regole, il considerevole numero di cittadini chiamati alle urne (quasi 9 milioni per le sole elezioni comunali), e l'importanza di alcune particolari arene come i comuni metropolitani di Milano, Torino e Catania. Anche se l'insieme delle comunità interessate non costituiva un vero e proprio campione rappresentativo dell'universo, la consultazione toccava una varietà di contesti geo-politici: dal nord-ovest (Novara, Vercelli, Pavia, Lecco) al nord-est (Belluno, Pordenone, Gorizia), dal centro (Ancona, Siena, Grosseto,

4 Decreto legislativo del 20-12-1993, n. 536 (collegi per la Camera).

5 Legge 10-12-1993, n. 515, Disciplina delle campagne elettorali per l'elezione alla Camera dei Deputati e al Senato della Repubblica. 
Terni) al Mezzogiorno (Agrigento, Cefalù, Vibo Valentia, Andria, Vasto $)^{6}$.

Dalla prova elettorale di giugno ci si attendeva la risposta a diversi quesiti che andavano al di là della pur importante scelta dei nuovi amministratori delle comunità interessate. In primo luogo, si trattava di capire come i diversi gruppi politici avrebbero interpretato $i$ vincoli $\mathrm{e}$ le opportunità offerte dal nuovo sistema e quanto rapido sarebbe stato l'apprendimento e l'adattamento alle rinnovate regole. In particolare il test poteva servire per misurare la capacità dei dirigenti politici di trovare, nella consultazione relativa ai sindaci ed ai presidenti delle province, formule di aggregazione appropriate alla nuova logica elettorale. Si trattava poi di vedere quali alleanze si sarebbero formate nelle città che per la loro importanza e visibilità sul piano nazionale costituivano veri e propri laboratori per la sperimentazione di formule alternative e dalle quali potevano venire indicazioni sulla evoluzione dei rapporti tra gruppi politici in ambiti più vasti in un non lontano futuro.

In secondo luogo, la tornata di elezioni amministrative costituiva un test importante sulle reazioni dell'elettorato sia rispetto agli aspetti tecnici delle nuove norme (voto separato per sindaco e consiglio, forte personalizzazione della contesa, eventuale doppio turno), sia a quelli più strettamente politici. In particolare il verdetto di giugno avrebbe fornito interessanti indicazioni sulla capacità di tenuta delle forze politiche tradizionali più direttamente toccate dalle rivelazioni emerse nelle varie inchieste giudiziarie sulle pratiche di finanziamento illecito dei partiti e sul coinvolgimento di personale politico in vari episodi di corruzione/concussione. Era presumibile, anche sulla base di alcuni mini-test elettorali locali svoltisi successivamente alle elezioni politiche del 1992, che i partiti tradizionalmente al governo avrebbero risentito negativamente degli sviluppi dell'azione della magistratura inquirente. Ma rimaneva incerta l'"entità" dei danni, in termini di consenso popolare, causati dalle rivelazioni di Tangentopoli dopo il 6 aprile del 1992. E agli effetti delle future prospettive delle formazioni di centro e, più in generale, del quadro politico, la «grandezza» del prezzo da pagare faceva una grossa differenza: un conto era ipotizzare un'ulterio-

${ }^{6}$ Le elezioni amministrative del 1993 sono oggetto di un ampio e ben documentato saggio di Aldo Di Virgilio (1994). 
re ma modesta erosione della base elettorale della compagine di centro-sinistra, tutt'altra cosa la prospettiva di un vero e proprio collasso. Correlativamente ci si poteva chiedere quale fosse l'ampiezza dell'onda di espansione del movimento leghista uscito trionfante $\mathrm{da}$ alcune consultazioni parziali nella seconda parte del 1992 e che le rilevazioni demoscopiche della primavera del 1993 davano in forte crescita in molte zone del nord del paese.

A fine giugno, tirando le somme dopo la tornata dedicata ai ballottaggi, risultava innanzitutto che non si era verificata quella riduzione dell'offerta che la nuova legge avrebbe dovuto incentivare. Il numero medio di liste presentate rimaneva infatti pressoché inalterato (circa 9) ed era di ben 10 il numero medio di candidature alla poltrona di sindaco ${ }^{7}$. Il cambiamento rispetto alle precedenti consultazioni amministrative riguardava piuttosto la forte riduzione del numero di liste recanti i contrassegni tradizionali dei partiti e il corrispettivo aumento di liste miste, civiche, di area, o anche di partito, ma caratterizzate da nuovi simboli a testimonianza degli sforzi di rinnovamento dei gruppi politici tradizionali già avviati in alcune comunità. Nei collegamenti tra liste e candidature per l'elezione del sindaco, l'offerta risultava frastagliata e differenziata da zona a zona anche dal punto di vista della composizione delle alleanze. Il fenomeno era particolarmente in evidenza nel settore di sinistra dello schieramento per la presenza di due principali configurazioni, quella del «fronte progressista» - una sinistra allargata comprendente tutti i gruppi di questo segmento, da Rifondazione ai Verdi - e quella della «sinistra-centro» che, mirando ai consensi del settore mediano dell'elettorato, escludeva dal cartello i neocomunisti e, in qualche caso, anche altri gruppi minori. Nel complesso, il panorama dell'offerta dava l'impressione di un quadro politico in movimento, caratterizzato da una certa fluidità, nel quale andavano facendosi più evidenti sia sintomi di una ulteriore de-strutturazione del mercato elettorale sia i primi segnali dell'inizio di un ciclo di ri-strutturazione.

Indicazioni convergenti provenivano dal verdetto emesso dagli elettori anche se la lettura dei risultati era resa difficile dalla particolare distribuzione territoriale delle arene interessate e dal fatto che non tutte le formazioni erano in gara negli stessi

${ }^{7}$ Di Virgilio $(1994,123)$. 
contesti. E tuttavia le variazioni intervenute nella forza elettorale dei diversi gruppi rispetto alla consultazione politica del 1992, riportate nella Figura 1, fornivano segnali piuttosto chiari sugli umori degli elettori. Vi era un chiaro declino complessivo dello schieramento di centro nel quale la tenuta o i modesti recuperi dei tre partners minori non bastavano a compensare le massicce perdite democristiane (un quinto della base elettorale Dc del 1992) e soprattutto il collasso del Psi che rispetto alla scadenza precedente perdeva quattro elettori su dieci. A ciò si accompagnavano una sostanziale stabilità della forza elettorale della destra e i modesti progressi dei gruppi di sinistra, leggermente più accentuati per Rete e Rifondazione. Ma il dato di fondo della tornata era rappresentato dal grande successo del movimento leghista emblematicamente rappresentato dalla conquista del comune di Milano, ma accompagnato anche da ottimi risultati in diversi altri contesti del nord del paese che portavano la Lega a raddoppiare la quota di consensi raccolti un anno prima ${ }^{8}$. Pur entro tutti i limiti rappresentati dalla parzialità della consultazione il test di giugno indicava una più accentuata propensione degli elettori, soprattutto di quelli moderati, a cambiare le loro opzioni di voto. Era un segnale che il «rimescolamento delle carte» di cui si vedevano ancora abbastanza timidi accenni a livello di élite avrebbe trovato, in presenza di una offerta adeguata, un riscontro nell'opinione pubblica.

Che i risultati del test di giugno non fossero una anomalia o il risultato di una particolare congiuntura veniva confermato a fine novembre da una seconda, importantissima tornata di elezioni amministrative per l'elezione di 424 consigli comunali (di cui 99 con popolazione superiore a 15.000 abitanti) dei consigli provinciali di Genova, Varese e La Spezia e del consiglio regionale del Trentino Alto Adige. Nel nuovo test, che chiamava alle urne oltre dieci milioni di elettori distribuiti anche in questo caso in diverse zone del territorio nazionale, assumevano particolare rilievo le consultazioni in sei grandi città (Roma, Napoli, Genova, Trieste, Venezia e Palermo) e in una dozzina di altri comuni capoluogo di provincia prevalentemente del centrosud $^{9}$.

${ }^{8}$ Nonostante l'esclusione del proprio candidato dal ballottaggio, la Lega risultava in testa alle preferenze degli elettori anche a Torino.

${ }^{2}$ Trattasi di: Alessandria, La Spezia, Macerata, Pescara, Chieti, Benevento, Taranto, Cosenza, Caltanisetta, Salerno, Caserta, Latina. 


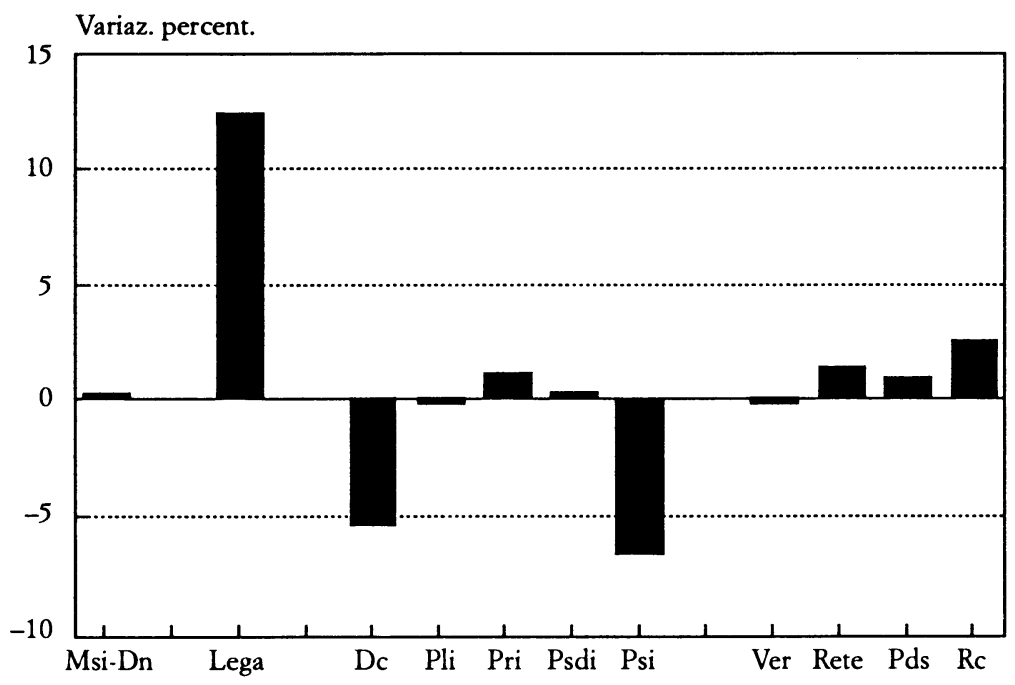

FIG. 1. Il declino del Centro. Aprile 1992-giugno 1993

La figura riporta le variazioni della percentuale di voti validi riportati dai diversi gruppi politici nelle elezioni politiche dell'aprile 1992 e nel primo turno delle elezioni per i consigli comunali del giugno 1993 nei comuni con oltre 15.000 abitanti interessati a questa tornata amministrativa. Va segnalato che il numero di comuni per $i$ quali è possibile il confronto tra le due consultazioni varia da partito a partito come segue: Dc (108), Pds (73), Rc (90), Msi-Dn (87), Psi (48), Rete (49), Lega (38), Psdi (35), Pri (27), Pli (6), Verdi (21).

Fonte: Servizio elettorale del Ministero degli Interni.

La consultazione costituiva una verifica dei risultati emersi nel test di giugno ma poteva anche essere considerata una sorta di prova generale delle future elezioni politiche che ormai si prevedevano a scadenza ravvicinata. Come, e forse più che a giugno, l'elemento di maggior interesse era costituito dalle possibili modifiche dei rapporti di forza tra i principali gruppi politici non tanto sul piano locale ma soprattutto a livello nazionale. In sostanza si trattava di capire chi avrebbe beneficiato del presumibile ulteriore arretramento delle formazioni di centro e, in particolare, quale fosse l'ulteriore capacità di espansione della Lega dopo la massiccia avanzata di giugno. Ad accentuare l'interesse degli osservatori contribuivano altri elementi: la verifica delle strategie di alleanza sperimentate nella tornata precedente; un prevedibile ulteriore incremento della personalizza- 
zione della contesa; la notorietà di alcuni protagonisti in duelli elettorali di particolare importanza (Rutelli e Fini a Roma, Bassolino e Mussolini a Napoli); la novità di alcune candidature scelte al di fuori della tradizionale nomenklatura come quelle dell'industriale del caffè Illy a Trieste, del filosofo Cacciari a Venezia, del magistrato Sansa a Genova, e di diversi candidati provenienti dalla società civile in altre comunità.

Il quadro delle alleanze presentava qualche novità rispetto alla prova di giugno. I gruppi politici che qualche mese prima avevano in molti casi affrontato da soli la competizione (Rifondazione, Msi-Dn e Dc), questa volta adottavano in un maggior numero di arene una strategia di cooperazione. Come è stato suggerito è probabile che questo cambiamento riflettesse, almeno in parte, un processo di graduale apprendimento intervenuto tra le due tornate ${ }^{10}$.

Dal computo dei voti ricevuti dalle liste scese in campo risultava con evidenza un nuovo arretramento, rispetto alle politiche del 1992, delle formazioni di centro, arretramento che toccava questa volta tutti e cinque i partiti (Figura 2). Particolarmente gravi erano le perdite subite dalla Dc la cui base veniva dimezzata e dal Psi che addirittura perdeva due elettori su tre. Il declino del centro già evidente a giugno riceveva dunque una piena conferma a novembre. A beneficiarne era ancora una volta il movimento leghista che anche in questa occasione raddoppiava i suoi consensi. Ma diversamente da quanto era accaduto a giugno, i risultati della seconda tornata evidenziavano anche un forte aumento dei voti a favore del Msi-Dn soprattutto nei comuni del centro-sud. A fronte di queste modifiche dei rapporti di forza al centro e a destra risultavano assai modeste le variazioni del voto sull'altro segmento dello schieramento nel quale accanto a sensibili progressi registrati dalle componenti minori (Verdi $+2,7 \%$ e Rete $+2,2 \%$ ) si riscontrava una sostanziale stabilità delle percentuali di voto per il Pds $(+0,7)$ e per Rifondazione $(+0,9)$.

Nonostante l'evidenza fornita da questi dati sulle quote di consenso ricevuto dalle varie liste, nei commenti post-elettorali finiva col prevalere una interpretazione del voto che metteva in risalto il grande successo delle sinistre. La cosa si spiega forse col fatto che l'attenzione degli osservatori era puntata prevalen- 


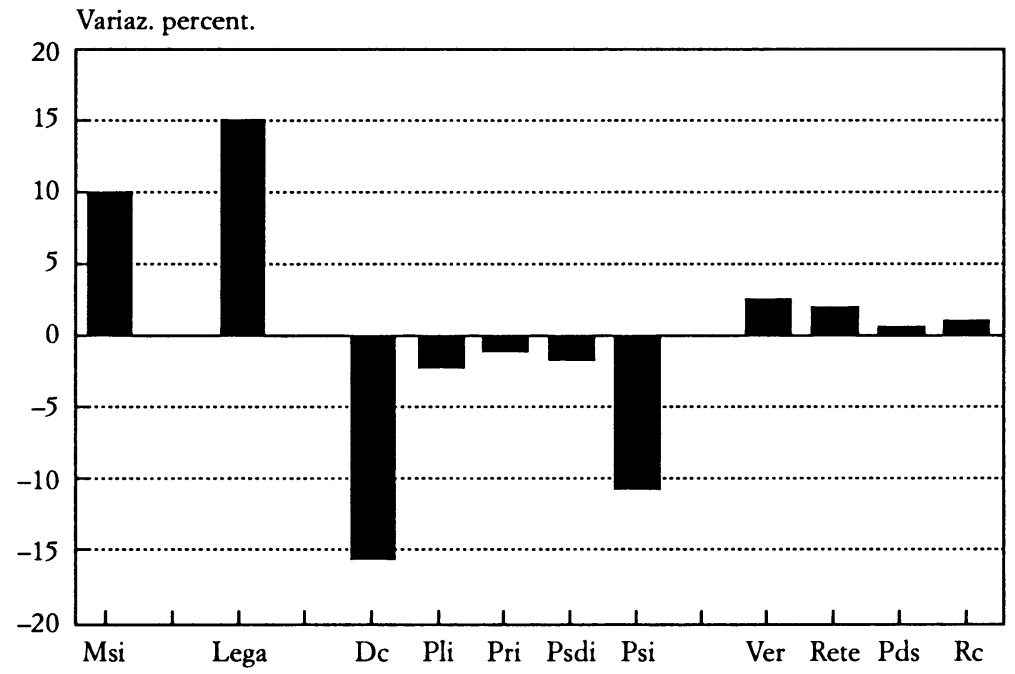

FIG. 2. Il declino del Centro. Aprile 1992-novembre 1993

La figura riporta le variazioni della percentuale di voti validi raggiunta dai diversi gruppi politici nelle elezioni politiche dell'aprile 1992 e nel primo turno delle elezioni per i consigli comunali del novembre-dicembre nei comuni con oltre 15.000 abitanti interessati a questa tornata amministrativa. Va segnalato che il numero di comuni per i quali è possibile il confronto tra le due consultazioni varia da partito a partito come segue: Dc (72), Pds (60), RC (73), Msi-Dn (62), Psi (13), Rete (36), Lega (25), Psdi (13), PRI (10), Pli (1), Verdi (35).

Fonte: Servizio elettorale del Ministero degli Interni.

temente sui ballottaggi del secondo turno per l'attribuzione delle cariche di sindaco, soprattutto di quelle delle grandi città. E le vittorie di Rutelli, Illy, Sanza, Cacciari e Bassolino (per non dire del trionfo di Orlando eletto a Palermo al primo turno) potevano certamente essere viste come prova della capacità delle alleanze progressiste di coagulare ampi consensi attorno a candidati comuni ${ }^{11}$. Ma si trattava di evidenze quantomeno parziali $e$ in una qualche misura fuorvianti. Infatti una lettura più attenta e più sistematica dei dati avrebbe dimostrato che gli esiti degli scontri per le cariche di sindaco si differenziavano ma non stravolgevano il senso dei risultati relativi al voto per le li-

11 Complessivamente i candidati progressisti appoggiati dal Pds erano risultati vincitori in 47 dei 99 comuni con oltre 15.000 abitanti. 
ste. Certo, guardando esclusivamente ai risultati di Trieste dove il candidato leghista era arrivato terzo e di Venezia e Genova dove i candidati leghisti erano stati sconfitti nei ballottaggi si poteva legittimamente concludere che l'avanzata della Lega era stata bloccata. Ma lo stesso non si poteva dire in molte altre arene medio-piccole in Lombardia, Piemonte, Veneto e Liguria nelle quali la Lega aveva conquistato il municipio ${ }^{12}$. Lo stesso può dirsi nel caso del Msi-Dn, decisamente fuori gioco al nord e sconfitto nei ballottaggi a Roma e a Napoli, ma capace di costituirsi come perno di alleanze vittoriose in oltre quaranta comuni del centro-sud tra i quali centri come Chieti, Latina, Benevento e Caltanisetta.

A ben vedere, la lezione che veniva dal test di dicembre era che se le forze di sinistra, e in particolare il Pds, avevano sviluppato meglio di altre la capacità di forgiare alleanze vincenti, la progressiva frana del centro tendeva a beneficiare il movimento leghista nelle regioni del nord e la destra in quelle del sud. Gli esiti delle due tornate amministrative del 1993 rafforzavano ulteriormente la frattura territoriale già prepotentemente emersa alle politiche dell'anno precedente contribuendo a farne un elemento rilevante per la dinamica politica nei mesi successivi soprattutto agli effetti della formazione delle alleanze elettorali.

\section{La destrutturazione del sistema dei partiti}

I risultati elettorali di giugno e di novembre erano una conferma dell'accresciuto dinamismo degli orientamenti di massa e comportavano sensibili modifiche dei rapporti di forza tra $i$ gruppi politici tradizionali. Ai segnali provenienti dalle urne si univano altre indicazioni sui cambiamenti in corso nella configurazione del sistema dei partiti. Tre sono gli elementi di questa evoluzione che meritano di essere sottolineati.

Il primo è costituito dal rapido disfacimento delle forze politiche che erano state per decenni alla guida del paese. Investiti dalla bufera di Tangentopoli i cinque partiti del centro-sinistra

12 Il gruppo di città e cittadine conquistate dal Carroccio comprende Alessandria, Acqui Terme, San Remo, Chiavari, Sestri Levante, Busto Arsizio, Legnano, Seregno, Lodi, Crema, Feltre, Montebelluna, Castelfranco Veneto, Chioggia e Jesolo. Nei comuni con meno di 15.000 abitanti, la Lega aveva ottenuto nel turno unico del 21 novembre ben 29 sindaci. 
erano già entrati in crisi nella seconda metà del 1992. Nel corso del 1993 col proseguimento e l'allargamento delle inchieste giudiziarie veniva inferto un altro gravissimo colpo alla leadership dei gruppi politici tradizionalmente al governo. L'evidenza del coinvolgimento di personaggi politici di primo piano in pratiche di finanziamento illecito dei partiti e in episodi di corruzione tanto ai vertici come in periferia si traduceva in un'ondata di discredito di queste forze agli occhi di una opinione pubblica già sospettosa, indignata e poco ben disposta nei confronti della classe politica in generale. Contemporaneamente venivano alla ribalta $\mathrm{i}$ problemi legati alla gestione di apparati di partito costosi e, nelle nuove circostanze, non più sostenibili. Ne derivava la necessità di una drastica riduzione delle macchine organizzative dei partiti sia al centro che in periferia con conseguente ulteriore perdita della capacità di raccogliere consensi. La crisi provocava inoltre confusione e sbandamento tra i militanti, turbati dalla scoperta di vicende e pratiche alle quali si sentivano estranei, col risultato di favorire ulteriormente fenomeni di smobilitazione della base già in corso per altre ragioni. Infine, era inevitabile che la tempesta abbattutasi su queste forze politiche creasse tensioni e fratture a livello dirigenziale tra il personale più direttamente compromesso dalle inchieste, naturalmente sulla difensiva, e $\mathrm{i}$ settori non direttamente investiti dagli scandali ma egualmente toccati, di riflesso, dalle conseguenze della crisi. Si veniva così a creare all'interno di queste forze politiche una frattura tra «conservatori» e «rinnovatori» che alimenterà nel corso dell'anno scontri e polemiche per sfociare alla fine in faticosi tentativi di ricostruzione e clamorose rotture.

Agli inizi del 1994, il bilancio dei partiti del vecchio centrosinistra era disastroso. Sparito di fatto il Psdi, sciolto il Pli, profondamente diviso il Pri sulla questione delle future alleanze elettorali, ridotto al lumicino il Psi in caduta verticale alle urne e dilaniato dai contrasti interni, usciva dalla scena come forza unitaria anche il partito che per mezzo secolo aveva avuto la maggioranza relativa. Ne raccoglievano l'eredità, divisi ed indeboliti, il Partito Popolare di Martinazzoli, in gestazione da mesi ma nato ufficialmente il 18 gennaio del 1994, e il Centro Cristiano Democratico cui aderivano una ventina di deputati e che teneva la sua prima assemblea sei giorni dopo.

La seconda componente era costituita dalla tenuta sul piano organizzativo ed elettorale, e su quest'ultimo anche con qualche progresso, delle forze tradizionalmente all'opposizione. Alla fine 
del 1993 sia Fini che Occhetto potevano guardare con un certo ottimismo alla prova elettorale ormai imminente. Per il Msi-Dn lo sfaldamento del vecchio centro rappresentava una grande opportunità per uscire dal ruolo di opposizione permanente che ne aveva caratterizzato la vita per decenni. L'estraneità del movimento dalle inchieste di Tangentopoli ne faceva un naturale beneficiario della perdita di consenso che aveva investito la classe politica coinvolta negli scandali. Il test elettorale d'autunno aveva inoltre dimostrato che il partito era in grado di proporsi con successo come perno di alleanze nelle quali potevano confluire, almeno nel mezzogiorno, pezzi del vecchio centro in disfacimento. La strada delle alleanze, un tempo del tutto impraticabile ma resa possibile dalla nuova congiuntura, diventava dunque la chiave per la fuoriuscita del partito dal ghetto dell'isolamento. Essa aveva anche il vantaggio di annacquare i collegamenti col neo-fascismo tramite l'utilizzazione di contrassegni in parte diversi da quelli del passato e poteva, quindi, servire a superare le residue diffidenze e le resistenze legate a questa connotazione. In questa logica è del tutto spiegabile il varo tra il dicembre del 1993 e il gennaio del 1994 di Alleanza Nazionale che si autodefiniva esplicitamente come formazione europea di centro-destra e nella quale confluivano, oltre al Msi-Dn, alcuni indipendenti e un nucleo di dirigenti della vecchia Dc.

Il $\mathrm{Pds}$, per quanto lambito, anche se in maniera relativamente marginale, dalle rivelazioni sugli episodi di corruzione, rimaneva pur sempre una forza rilevante (e tanto più appariva esserlo in un panorama politico crescentemente frammentato) ed un elemento indispensabile per la costruzione di ogni alleanza sul versante di sinistra dello schieramento sia per le formule «progressiste» aperte verso il centro sia per le soluzioni di sinistra più tradizionali. Vi erano però anche degli elementi che suscitavano qualche dubbio sulle prospettive future: la modesta performance delle liste pidiessine nei test di giugno e novembre, la presenza sul fianco sinistro di un potenziale alleato-rivale (Rifondazione) che avrebbe reso problematiche le aperture verso il centro ma del quale era difficile fare a meno, e il peso di essere agli occhi di una parte dell'opinione pubblica l'erede del vecchio partito comunista.

La terza modifica alla tradizionale configurazione del sistema dei partiti era rappresentata dall'affacciarsi alla ribalta di forze nuove. Alcune di queste si possono considerare nuove rispetto alla schieramento tradizionale, ma non nuovissime. $\grave{E}$ il 
caso di Lega Nord e Rete, già presenti nell'arena politica nel 1992 e che si rafforzavano, anche se evidentemente in misura ben diversa, nel corso del 1993. Altri gruppi, come Alleanza Democratica e Patto Segni, erano venuti alla ribalta dopo la consultazione politica di quell'anno ed erano stati promotori attivi della campagna per la riforma elettorale. Agli inizi del 1994 tutte e quattro queste formazioni erano tra i protagonisti del dibattito politico e giocavano un ruolo rilevante nelle schermaglie sugli schieramenti che si andavano formando, un ruolo in alcuni casi sproporzionato rispetto al loro potenziale elettorale.

$\mathrm{Ma}$ la novità più importante agli effetti della configurazione del sistema partitico era costituita dall'emergere, a cavallo tra la fine del 1993 e l'inizio del 1994, di un'entità del tutto nuova, il movimento «Forza Italia» creato per iniziativa di Berlusconi con utilizzazione di risorse organizzative e personale di aziende del gruppo Fininvest. Dico «entità» e non gruppo politico perché sulla natura del movimento, di cui inizialmente veniva smentita addirittura la costituzione, permanevano a lungo molti dubbi, quantomeno all'esterno. Collegata con una «Associazione per il Buongoverno», anch'essa al debutto, Forza Italia veniva dapprima presentata come un movimento di opinione organizzato in una rete di clubs che si proponeva di influenzare l'evoluzione del quadro politico in una fase di transizione ma senza un diretto coinvolgimento nell'arena elettorale. In una seconda fase, quando filtravano evidenti segnali di propositi diversi da quelli inizialmente indicati (come, ad esempio, la ricerca e la selezione di potenziali candidati o i sondaggi sulla popolarità di Berlusconi), la possibilità che Forza Italia si trasformasse da movimento in partito non veniva esclusa ma neppure confermata. In una terza fase, in presenza di crescenti indizi e di esplicite ammissioni sulla natura politica del movimento, il possibile ruolo di Forza Italia era, e veniva usato, come una sorta di "spada di Damocle" appesa sopra la testa degli altri protagonisti e ne complicava le mosse tattiche. Considerate le sue risorse sul piano organizzativo e su quello dei mezzi di comunicazione e la sua capacità di attrarre consensi comprovata dalle rilevazioni demoscopiche, un intervento diretto di Berlusconi nella consultazione avrebbe decisamente influito sulle prospettive elettorali di altri competitori ${ }^{13}$.

13 A metà gennaio 1994 i sondaggi condotti da istituti esterni alla Fininvest accreditavano Forza Italia di un $12 \%$ delle intenzioni di voto espresse dagli intervistati. 


\section{Il rebus delle alleanze}

All'inizio del 1994 tutto era pronto dal punto di vista istituzionale-procedurale per l'elezione di un nuovo Parlamento che ormai si annunciava come imminente anche se rimanevano non poche resistenze ad un rapido scioglimento delle camere e molte incertezze sulla data. Tuttavia, contrariamente a quanto era avvenuto in passato, a poche settimane dal voto dal punto di vista degli schieramenti che sarebbero scesi in campo la situazione permaneva assai fluida: era assai difficile dire come i protagonisti - vecchi, nuovi e rinnovati - avrebbero affrontato lo scontro. E il quadro era destinato a rimanere tale anche dopo la decisione presa a metà gennaio dal capo dello Stato di sciogliere il Parlamento ed indire le elezioni per la fine di marzo.

La fluidità del quadro dipendeva in parte dalla presenza di alcuni attori politici del tutto nuovi, o almeno rinnovati, e in parte dalla sperimentazione di meccanismi elettorali mai messi alla prova. I vincoli e le opportunità offerti dal nuovo sistema elettorale producevano spinte in parte contraddittorie: da un lato le regole per la competizione nei collegi uninominali costituivano una forte incentivo a cercare forme di aggregazione; dall'altro, l'esistenza della quota di seggi da attribuire in ragione proporzionale non scoraggiava le spinte centrifughe e favoriva il mantenimento della distinta identità di ciascuno dei gruppi politici. Oltre a queste spinte contraddittorie provenienti dalle regole altri fattori rendevano assai improbabile la fusione o la formazione di solide alleanze nel poco tempo a disposizione: le ambizioni personali, le radicate preclusioni, gli interessi e le identità di gruppo. Date queste circostanze, il risultato più probabile era la presentazione di liste separate nelle circoscrizioni e la stipulazione di accordi ad boc su candidature comuni nei collegi, accordi accompagnati da un piano di ripartizione (o di spartizione) dei collegi da assegnarsi ai partners di ciascun cartello in proporzioni da definire.

Alla conclusione delle trattative emergevano tre schieramenti: quello dei «Progressisti» (comprendente Pds, Rifondazione, Rete, Verdi, Alleanza Democratica, Cristiano-Sociali e Partito Socialista), quello di Centro (Partito Popolare e Patto Segni) e un cartello elettorale di destra articolato nelle due sotto-alleanze denominate «Polo delle Libertà» (Forza Italia, Lega Nord, Centro Cristiano Democratico, Unione di Centro) e «Polo del Buongoverno» (Forza Italia, Alleanza Nazionale, Ccd e Uc). In 
quest'ultimo caso si trattava di uno schieramento particolare per varie ragioni. Intanto per la presenza nei due cartelli di partners comuni ( $\mathrm{ma}$ in particolare del movimento di Berlusconi) che teneva insieme due «alleati» difficilmente compatibili, anzi palesemente l'un l'altro ostili. Poi per gli accordi sulla differenziazione su basi geografiche delle candidature che prevedevano la presenza di un polo nelle regioni e circoscrizioni del nord e dell'altro in quelle del Mezzogiorno. E, infine, per il fatto che Alleanza Nazionale non aveva rinunciato a presentare candidati propri nel centro-nord col risultato che in queste zone si trovava a competere con gli altri due partners. Era una soluzione piuttosto singolare resa possibile dal diverso insediamento territoriale della Lega e di Alleanza Nazionale le cui reciproche preclusioni venivano attenuate dal ruolo di cuscinetto di Forza Italia.

La cronaca delle giornate che precedevano la scadenza per la presentazione dei simboli ( 15 febbraio) e delle candidature (21 febbraio), era punteggiata da incontri, scontri e convulse trattative che investivano tutti o quasi i segmenti dello spettro politico. Per ovvie ragioni, è impossibile dar conto in questa sede di tutte le mosse e contro-mosse che hanno caratterizzato la fase immediatamente precedente all'inizio vero e proprio della campagna elettorale. Tra l'altro, molte trattative si sono svolte al riparo di occhi indiscreti e le circostanze nelle quali sono state prese importanti decisioni difficilmente possono essere conosciute da un osservatore esterno. Lo stesso dicasi per il ruolo giocato dalle informazioni disponibili per alcuni protagonisti, ma non per tutti, sull'evolversi degli orientamenti dell'opinione pubblica o delle percezioni delle mosse degli altri attori. Non v'è dubbio, in ogni caso, che si sia trattato di una fase intensa di rapporti tra gruppi politici caratterizzata da colpi di scena, avvicinamenti e rotture, aperture e chiusure, come indica il sintetico riepilogo della tabella 1.

Al di là dei singoli episodi, ripercorrendo le tappe di questa vicenda tentando di calarsi nell'atmosfera del momento, si possono ricavare alcune osservazioni di carattere più generale qui sotto elencate senza alcune pretese di completezza.

1. Fino alle scadenze fissate per legge vi è stata grande incertezza, probabilmente negli stessi protagonisti e certamente negli osservatori, sugli esiti dei complessi processi di decisione in merito agli accordi elettorali. Contrariamente a quanto forse si potrebbe pensare a posteriori, la partita delle alleanze è rimasta aperta fino all'ultimo. 
TAB. 1. Trattative per le alleanze elettorali nei primi mesi del 1994.

17-1 - Berlusconi rinvia la sua intervista al TG-1 nella quale doveva annunciare le sue intenzioni.

19-1 - Conferenza stampa di Berlusconi che dà un «ultimatum» alle forze moderate per la costituzione di una alleanza elettorale che dovrebbe comprendere Ppi, Lega Nord e Patto Segni minacciando in caso contrario di scendere in campo alla testa di Forza Italia.

- Apertura del Pds e di Ad al Partito Popolare.

22-1 - Incontro tra Segni e Martinazzoli in merito ad una eventuale apertura alla Lega.

- Mastella del Ccd non esclude accordi elettorali col Msi.

23-1 - All'assemblea del Ppi Martinazzoli ribadisce l'indisponibilità del gruppo ad alleanze con Pds, Lega e Forza Italia.

- Alla cerimonia di presentazione di Alleanza Nazionale aperture di Fini a Forza Italia e Lega.

25-1 - Annuncio di un accordo elettorale tra Patto Segni e Lega.

- Preclusioni della Rete all'ingresso di socialisti e repubblicani nell'alleanza di sinistra.

26-1 - Sfuma l'accordo tra la Lega e il Patto per i veti di Bossi e del Ppi.

- Messaggio televisivo registrato col quale Berlusconi annuncia l'ingresso di Forza Italia nell'arena elettorale.

- Pds, Ad e Cristiano Sociali siglano un primo accordo sul programma elettorale.

29-1 - Al Congresso del Msi emergono ipotesi di accordo con Forza Italia e il Ccd.

- Nuova proposta di Occhetto al Ppi respinta da Martinazzoli.

30-1 - L'assemblea socialista sceglie un nuovo simbolo e opta per entrare nell'alleanza di sinistra.

31-1 - Rottura nel PRI sulla scelta dello schieramento: 14 parlamentari in dissenso con la linea di La Malfa.

1-2 - Primo annuncio di un «quasi accordo» tra Lega e Forza Italia, ma continuano le polemiche tra Bossi e Berlusconi.

2-2 - Firmato l'accordo tra gli otto gruppi che compongono il «cartello progressista».

4-2 - Berlusconi non esclude che Forza Italia possa competere senza la Lega.

- Affiorano subito tensioni all'interno dell'area progressista.

5-2 - Al congresso della Lega Bossi annuncia il patto elettorale con Forza Italia ma esclude ogni apertura ad Alleanza Nazionale.

6-2 - Prima manifestazione ufficiale di Forza Italia con discorso di un'ora trasmesso integralmente da una delle reti Fininvest.

- Via libera della Lega ad accordi tecnici tra Forza Italia e Alleanza Nazionale nel centro-sud.

7-2 - Decisa chiusura della Lega ad Alleanza Nazionale («Mai con i fascisti»).

8-2 - Polemiche sulla scelta dei candidati all'interno del fronte progressista.

9.2 - Polemiche sulla scelta dei candidati tra Popolari e Pattisti.

11-2 - Accordo tra Berlusconi e Bossi sulle candidature al Nord e tra Forza Italia e Alleanza Nazionale per i collegi del centro-sud.

12-2 - Ufficializzato l'accordo elettorale tra Forza Italia, Lega, Ccd e Unione di Centro.

14-2 - Forti tensioni tra Popolari e Pattisti sull'inclusione tra i candidati di politici inquisiti. 
2. A mantenere alto il grado di incertezza ha contribuito il rimescolamento delle carte dovuto al rinnovamento di alcuni gruppi politici, ma soprattutto l'incognita legata all'entrata in campo di una forza politica del tutto nuova, con un potenziale elettorale sconosciuto e che, almeno formalmente, si è schierata solo all'ultimo momento.

3. I cartelli elettorali che alla fine sono emersi dal valzer degli incontri tra i leaders erano solo alcune delle soluzioni politicamente possibili e non l'unico, inevitabile e predeterminato sbocco dei rapporti tra le forze in campo. Ciò sottolinea, ancora una volta, l'importanza delle scelte effettuate dai dirigenti dei gruppi in competizione soprattutto in contesti relativamente poco strutturati e nei quali nuove regole incentivano comportamenti di tipo «cooperativo» e la ricerca di forme di aggregazione.

4. La composizione dei tre cartelli elettorali emersi dai negoziati smentisce almeno in parte la tesi di un presunto declino della dimensione sinistra-destra come asse portante della competizione elettorale. Nonostante le novità, le alleanze non si discostano molto dalle tradizionali «famiglie» politiche del passato. Inoltre gli ostacoli e gli accordi, sia quelli fatti che quelli mancati, testimoniano la persistenza di un forte radicamento delle immagini (o auto-immagini) dei protagonisti nel tradizionale continuum.

5. Le alterne vicende dei negoziati rivelano la presenza di pressioni contrastanti provenienti da un lato dagli incentivi del sistema maggioritario ad evitare l'isolamento e, dall'altro, dalla persistenza di forti preclusioni e steccati radicati nelle esperienze del passato.

6. La ricerca di partners accettabili ha generato tensioni all'interno dei singoli gruppi politici. Queste hanno avuto ad oggetto in alcuni casi la scelta dello schieramento nel quale confluire e/o la scelta delle persone da ammettere tra i candidati.

7. Durante le trattative, come anche dopo il raggiungimento di accordi di massima, si sono sviluppate tensioni tra i partners delle coalizioni sulla quota di candidati comuni provenienti dai diversi gruppi nonché sulla scelta dei collegi da destinare loro o sulla posizione dei candidati nelle liste di circoscrizione.

8. Le problematiche di cui ai punti precedenti hanno spesso generato frizioni anche tra centro e periferia. Questioni genericamente risolte dai leaders negli accordi a livello nazionale sono puntualmente riaffiorate quando le trattative si sono spo- 
state dal centro ai «tavoli regionali» per le scelte definitive sulle candidature.

\section{Gli elettori verso il voto}

La definizione delle regole e la formazione degli schieramenti rimuovevano due dei principali fattori di incertezza. Rimaneva da vedere quale sarebbe stata la risposta dei cittadini ai programmi ed alle candidature proposte dagli schieramenti in campo nel contesto del nuovo sistema elettorale.

Un primo gruppo di interrogativi riguardava la conoscenza e le modalità di utilizzazione delle nuove regole da parte degli elettori. Di particolare interesse, da questo punto di vista, era l'innovazione della doppia scheda per l'elezione dei deputati e la possibilità offerta ai cittadini di votare in maniera difforme per le candidature del collegio e le liste di circoscrizione. Era presumibile che le ridotte dimensioni dei collegi e il numero contenuto di candidati avrebbero fortemente personalizzato le battaglie nei 475 collegi ma rimaneva da vedere quali ne sarebbero state le conseguenze agli effetti del doppio voto. Tra l'altro, la presenza nel proprio collegio di un candidato non gradito, ancorché presentato dallo schieramento preferito, avrebbe potuto indurre qualche elettore ad esprimere solo il voto di lista. Altre incognite di questo tipo riguardavano la conoscenza da parte degli elettori della clausola di esclusione fissata al $4 \%$ e dei suoi effetti ai fini dell'assegnazione dei seggi. Per i gruppi politici minori, che avevano deciso di correre il rischio di presentarsi separatamente nelle circoscrizioni, le percezioni dell'elettorato sulla probabile forza dei gruppi e sugli effetti della clausola potevano avere un'influenza decisiva.

Un secondo interrogativo riguardava il ruolo che avrebbero avuto sulla formazione delle scelte degli elettori i mezzi di comunicazione di massa e, in particolare, la televisione sia attraverso i canali a diffusione nazionale sia attraverso quelli locali. Ad accrescere l'interesse per questo tema contribuivano, ovviamente, i legami tra Forza Italia e le tre reti Fininvest, ma anche altri elementi come i limiti posti alla trasmissione di spot e le modalità di applicazione della nuova disciplina delle campagne elettorali, la preannunciata personalizzazione della contesa che avrebbe reso il mezzo televisivo un efficace strumento per la diffusione di immagini positive dei candidati, le ridotte dimen- 
sioni dei collegi che potevano aprire ampi spazi alle emittenti locali.

$\mathrm{Al}$ ruolo di questi fattori era collegato l'interrogativo principale della consultazione, quello relativo ai comportamenti dei settori di centro dell'elettorato. Un'emorragia di consensi come quella verificatasi nel corso del 1993 avrebbe costituito un duro colpo per la speranza dell'alleanza di Popolari e Pattisti di ricoprire il ruolo di ago della bilancia nel nuovo Parlamento. Si trattava poi di vedere in quale direzione si sarebbero eventualmente spostati gli elettori che un tempo avevano privilegiato la Dc, il Psi ed i tre partiti laici. Flussi non equilibrati di questi elettori nell'una o nell'altra direzione avrebbero avuto un'influenza decisiva sulle sorti del fronte progressista e dei due poli del centro-destra.

Quest'ultimo interrogativo avrebbe trovato una risposta definitiva nel conteggio dei voti alla fine di marzo, ma alcuni segnali sull'esito della consultazione erano già molto evidenti nelle rilevazioni demoscopiche effettuate nella fase precedente l'inizio ufficiale della campagna (Fig. 3). Pochi giorni dopo lo scioglimento delle camere Lega Nord, Alleanza Nazionale ed il movimento di Berlusconi (non ancora ufficialmente in campo) potevano già contare su oltre un terzo delle intenzioni di voto dichiarate dagli elettori intervistati dall'istituto Cirm, mentre lo schieramento progressista era dato al $40 \%$ e Popolari e Pattisti insieme superavano di poco il $20 \%$. Il declino del centro era dunque confermato ma non era ancora chiaro a vantaggio di chi. Nel corso delle due successive settimane, dopo la formale dichiarazione di ingresso nell'arena di Forza Italia, avveniva prima il sorpasso e poi un allargamento della forbice tra $i$ due principali competitori mentre il centro perdeva ulteriori consensi. A fine febbraio, quando partiva ufficialmente la campagna, le intenzioni di voto manifestate dai cittadini mostravano che le quote di consenso raccolte dai tre gruppi concorrenti non si discostavano molto da quello che sarebbe poi stato l'esito finale della competizione. Questa constatazione suggerisce che la cristallizazione delle scelte possa essere avvenuta in larga misura durante la pre-campagna quando non era ancora applicabile la disciplina prevista dalle nuove norme, in particolare quelle relative all'utilizzazione dei messaggi televisivi.

Ma questa è solo una delle possibili ipotesi che si possono fare circa i fattori che hanno influito sulla formazione delle scelte degli elettori. Sui «come», «quando», «dove» e «perché» del 


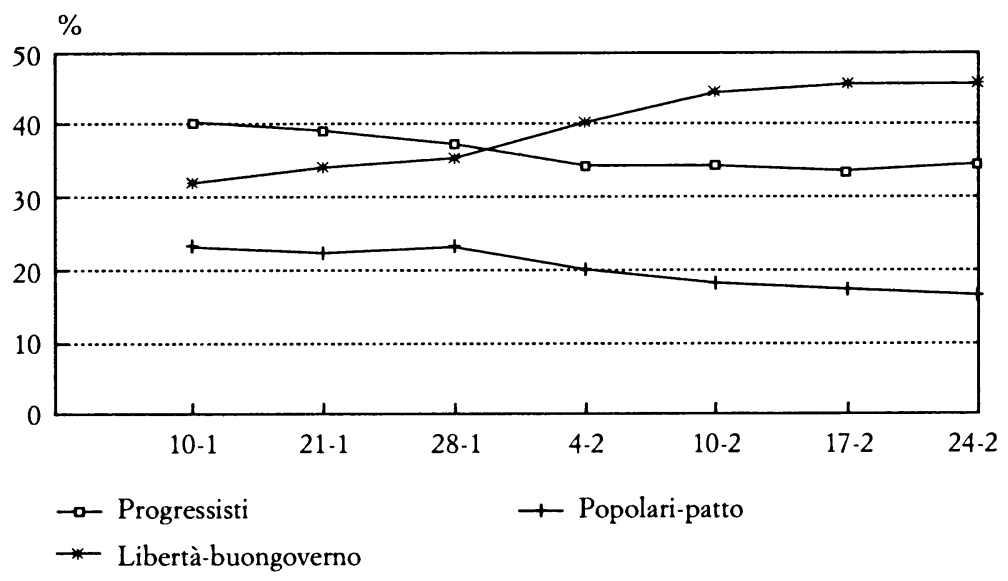

FIG. 3. Intenzioni di voto. Gennaio-febbraio 1994

Il grafico è basato sui risultati di alcuni sondaggi pre-elettorali condotti dall'istituto Cirm per Telemontecarlo e pubblicati su «La Repubblica» alle date indicate. Le intenzioni di voto espresse per $\mathrm{i}$ singoli gruppi politici sono state aggregate per schieramento.

verdetto il lettore troverà più ampie ed approfondite analisi negli altri contributi di questo stesso numero della rivista.

\section{Riferimenti bibliografici}

Camera dei Deputati (1994), Norme per l'Elezione della Camera e del Senato, Roma, Quaderni di documentazione del Servizio Studi.

D'Alimonte, R. e A., Chiaramonte (1993), Il nuovo sistema elettorale italiano: quali opportunità, in «Rivista Italiana di Scienza Politica», XXIII, pp. 513-547.

Di Virgilio, A. (1994), Elezioni locali e destrutturazione partitica. La nuova legge alla prova, in «Rivista Italiana di Scienza Politica», XXIV, pp. 107-166. 